\title{
Effect of maternal dyslipidaemia on the cardiorespiratory physiology and biochemical parameters in male rat offspring
}

\author{
Keyth Sulamitta L. Guimarães ${ }^{1}$, Emmanuel Veríssimo de Araújo ${ }^{1}$, Jailane Souza Aquino ${ }^{1}$, \\ Danilo Assis Gadelha ${ }^{2}$, Camille Moura Balarini ${ }^{2,3}$, João Henrique Costa-Silva ${ }^{4}$, Marciane Magnani ${ }^{5}$, \\ Hubert $\mathrm{Vidal}^{6}$, Valdir Andrade Braga ${ }^{2}$ and José Luiz de Brito Alves ${ }^{1,6_{*}}$ \\ ${ }^{1}$ Department of Nutrition, Health Sciences Center, Federal University of Paraiba, Joao Pessoa, 58051-900, Brazil \\ ${ }^{2}$ Biotechnology Center, Federal University of Paraiba, Joao Pessoa, 58051-900, Brazil \\ ${ }^{3}$ Department of Physiology and Pathology, Health Sciences Center, Federal University of Paraiba, Joao Pessoa, 58051-900, Brazil \\ ${ }^{4}$ Department of Physical Education and Sport Sciences, Federal University of Pernambuco, Vitória de Santo Antão - PE, \\ 55608-680, Brazil \\ ${ }^{5}$ Department of Food Engineering, Technology Center, Federal University of Paraiba, Joao Pessoa, 58051-900, Brazil \\ ${ }^{6}$ CarMeN (Cardio, Metabolism, Diabetes and Nutrition) Laboratory, Institut National de la Santé et de la Recherche Médicale \\ (INSERM) U1060, Institut National de la Recherche Agronomique (INRA) U1397, Institut National des Sciences Appliquées \\ (INSA) Lyon, Université Claude Bernard Lyon 1, Univ-Lyon, 69600 Oullins, France
}

(Submitted 27 June 2017 - Final revision received 24 August 2017 - Accepted 21 September 2017)

\section{Abstract}

The present study evaluated the effects of maternal dyslipidaemia on blood pressure (BP), cardiorespiratory physiology and biochemical parameters in male offspring. Wistar rat dams were fed either a control (CTL) or a dyslipidaemic (DLP) diet during pregnancy and lactation. After weaning, both CTL and DLP offspring received standard diet. On the 30th and 90th day of life, blood samples were collected for metabolic analyses. Direct measurements of $\mathrm{BP}$, respiratory frequency $\left(\mathrm{R}_{\mathrm{F}}\right)$, tidal volume $\left(\mathrm{V}_{\mathrm{T}}\right)$ and ventilation $\left(\mathrm{V}_{\mathrm{E}}\right)$ under baseline condition, as well as during hypercapnia $\left(7 \% \mathrm{CO}_{2}\right.$ ) and hypoxia (KCN, $0.04 \%$ ), were recorded from awake 90-d-old male offspring. DLP dams exhibited raised serum levels of total cholesterol (TC) (4·0-fold), TAG (2.0-fold), VLDL + LDL (7·7-fold) and reduced HDL-cholesterol (2.4-fold), insulin resistance and hepatic steatosis at the end of lactation. At $30 \mathrm{~d}$ of age, the DLP offspring showed an increase in the serum levels of TC $(P<0 \cdot 05)$ and VLDL $+\mathrm{LDL}$ $(P<0.05)$ in comparison with CTL offspring. At $90 \mathrm{~d}$ of age, DLP offspring exhibited higher mean arterial pressure (MAP, approximately $34 \%)$. In the spectral analysis, the DLP group showed augmented low-frequency (LF) power and LF:high-frequency (HF) ratio when compared with CTL offspring. In addition, the DLP animals showed a larger delta variation in arterial pressure after administration of the ganglionic blocker $(P=0.0003)$. We also found that cardiorespiratory response to hypercapnia and hypoxia was augmented in DLP offspring. In conclusion, the present data show that maternal dyslipidaemia alters cardiorespiratory physiology and may be a predisposing factor for hypertension at adulthood.

\section{Key words: Maternal dyslipidaemia: Biochemical parameters: Hypertension: Chemosensitivity}

Arterial hypertension and metabolic disorders remain the main risk factors for cardiovascular diseases and are associated with high morbidity and mortality rates worldwide ${ }^{(1,2)}$. This indicates that a better understanding of the underlying mechanisms causing hypertension and metabolic impairment is essential for effective interventions and prevention strategies.

The excessive consumption of fat and sugar, usually described as the westernised lifestyle, has contributed to the growing incidence of metabolic disorders such as dyslipidaemia and type 2 diabetes $^{(3)}$. It has been established that metabolic disorders, especially during pregnancy and early life, enhance susceptibility to the development of cardiometabolic diseases ${ }^{(4-7)}$. For example, offspring from dams exposed to a high-fat diet during pregnancy exhibit hypertension and metabolic alterations in adult life ${ }^{(8,9)}$.

Maternal dyslipidaemia is a serious metabolic disorder with a limited therapeutic approach. It has been described as an important risk factor for fetal death, preterm birth and with adverse effects on offspring development ${ }^{(10,11)}$. Increasing evidence suggests that a maternal dysmetabolic condition, such as maternal dyslipidaemia, may compromise placental functionality and impair the fetus-placental supply through elevated oxidative stress ${ }^{(12)}$, pro-inflammatory markers ${ }^{(13)}$ and altered lipid and glucose handling ${ }^{(14)}$.

Abbreviations: ECG, electrocardiogram; HF, high frequency; HR, heart rate; ITT, insulin tolerance test; LF, low frequency; MAP, mean arterial pressure; MDA, malondialdehyde; OGTT, oral glucose tolerance test; RF, respiratory frequency; TC, total cholesterol; VE, ventilation; VT, tidal volume.

* Corresponding author: J. L. de Brito Alves, email jose_luiz_61@hotmail.com 
Previous studies have shown that the increase in proinflammatory markers and imbalance in oxidative stress can alter the mechanisms of blood pressure (BP) control, such as: (i) decrease in baroreflex sensitivity ${ }^{(15)}$, (ii) respiratory chemosensitivity dysregulation ${ }^{(16-18)}$ and (iii) sympathetic hyperactivity ${ }^{(19,20)}$. It has been also established that elevated sympathetic activity is involved in the generation of hypertension ${ }^{(21,22)}$ and is associated with metabolic disorders ${ }^{(23-25)}$.

It is reported that the sympathetic nervous system may be modulated via central and peripheral respiratory chemoreceptors, evoking reflex responses of sympatho-excitation and tachypnoea ${ }^{(26,27)}$. However, it remains unknown whether a sympathetic hyperactivity, in part modulated by raised respiratory chemosensitivity, could be a potential mechanism underlying maternal dyslipidaemia-induced hypertension and metabolic disorders.

Thus, in the present study, we examined the effects of maternal dyslipidaemia during pregnancy and lactation on the cardiorespiratory physiology and glucose-lipid biochemical parameters in male offspring. Our hypothesis was that a sympathetic hyperactivity associated with higher respiratory chemosensitivity could be a potential hidden basis for the development of arterial hypertension and metabolic disorder in adult male offspring from dams exposed to a dyslipidaemic (DLP) diet during pregnancy and lactation.

\section{Methods}

Experimental procedures were performed in accordance with the revised guide for the care and use of laboratory animals ${ }^{(28)}$. The experimental protocol was submitted and approved by the institutional animal care and use committee (CEUA-UFPB protocol 014/2016) of the Federal University of Paraiba, Brazil.

\section{Animals and experimental design}

In all, ten virgin female Wistar rats (Rattus norvegicus) were maintained at room temperature $\left(22 \pm 1^{\circ} \mathrm{C}\right)$ with a controlled light-dark cycle (dark 06.00-18.00 hours). A standard laboratory chow diet (52\% carbohydrate, $21 \%$ protein and $4 \%$ lipids; Purina Agriband) and water were given ad libitum. At $90 \mathrm{~d}$ of age, rats were subjected to dietary manipulations: control (CTL group, $n$ 5) and DLP diets (DLP group, $n$ 5) were offered ad libitum during 1 week. Then, the rats were mated in a special cage overnight (one female for one male) and the pregnant females were transferred to individual cages. The CTL diet was prepared according to the American Institute of Nutrition ${ }^{(29,30)}$ and the DLP diet was purchased from Rhoster ${ }^{\circledR}$ Company. The diets (Table 1) were offered during the pregnancy and lactation periods. At $24 \mathrm{~h}$ after birth, the offspring were randomly adjusted to eight pups (four male and four female) per litter to ensure standardised nutrition until weaning. At weaning (postnatal day 22), the female and male offspring were housed separately (four per cage) and had free access to a normal diet until the beginning of the experimental phase. The CTL and DLP experimental groups were formed with two rats from each litter. We first examined the impact of maternal dyslipidaemia on serum glucose and lipid parameters in 30-dold rats. Then, the male rats were maintained in the protocol to
Table 1. Nutritional composition of the diets $(\mathrm{g} / 100 \mathrm{~g}$ diet)

\begin{tabular}{lcc}
\hline Nutrients & Control diet (AIN-93G) & Dyslipidaemic diet \\
\hline Casein (85\%) & 20 & 19.86 \\
Dextrin maize starch & 13 & 15.5 \\
Cellulose & 5 & 5 \\
Sucrose & 10 & 6 \\
Maize starch & 39.7 & 33.6 \\
Soyabean oil & 7 & 3 \\
Animal fat (lard) & 0 & 6 \\
Non-hydrolysed vegetable fat & 0 & 5 \\
Gallic acid (Sigma) & 0 & 0.5 \\
Cholesterol (Sigma) & 0 & 0.5 \\
Choline & 0.25 & 0.25 \\
Methionine & 0.3 & 0.3 \\
Vitamin mix & 1 & 1 \\
Mineral mix & 3.5 & 3.5 \\
Tert-butylated hydroxytoluene & 0.014 & 0.014 \\
\hline
\end{tabular}

* Casein showed $85 \%$ purity ( $85 \mathrm{~g}$ protein for each $100 \mathrm{~g}$ casein).

access the long-term effect of maternal dyslipidaemia on biochemical parameters and cardiorespiratory physiology at day 90. Female offspring were not included in the present study.

\section{Effects of the dyslipidaemic diet during pregnancy and lactation in dams}

From the 1st day of pregnancy to the end of the lactation, the body weight and food intake of each dam were measured daily using a digital scale (model AS-1000; Marte) having an error range of $0 \cdot 1 \mathrm{~g}$. Food intake was monitored by measuring the difference between the amount of food put in the cage and the amount remaining after $24 \mathrm{~h}$. The results of body weight and food consumption were compiled and expressed as weekly consumption.

At $24 \mathrm{~h}$ after weaning, the dams were fasted overnight and then an oral glucose tolerance test (OGTT) was performed. Then, $24 \mathrm{~h}$ after the OGTT, the dams were again fasted overnight and an insulin tolerance test (ITT) was performed after an intraperitoneal injection of insulin $(0.75 \mathrm{IU} / \mathrm{kg}$ body weight). Blood glucose concentrations were measured before ( $0 \mathrm{~min}$ ) and after $(30,60,90$ and $120 \mathrm{~min}$ ). Last, $48 \mathrm{~h}$ after the ITT, the dams were fasted overnight and euthanised by decapitation. The dams had remained on a DLP diet during these four days after weaning. Serum samples were collected for quantification of total cholesterol (TC), TAG and HDL with commercial kits (Bioclin). The LDL and VLDL values were calculated by the use of previously described formulas ${ }^{(31)}$.

The livers from both groups were collected for analysis of lipid accumulation. Liver tissue was excised, washed in saline solution $(0.9 \% \mathrm{NaCl})$ and fixed in buffered formalin $(10 \%)$ for $48 \mathrm{~h}$. Then, the tissues were sectioned in microtome to obtain 8 - $\mu$ m-thick slices. A total of ten sections were stained with Oil Red O (Sigma) for $10 \mathrm{~min}$. Sections were examined under light microscopy $(4 \times$ and $20 \times$ ) and representative photos were acquired for each group $^{(32)}$. Another ten different sections were also stained with haematoxylin-eosin and analysed under a common light microscope (Motic BA 200; Scientific Instrument Company).

\section{Measurement of somatic parameters in male offspring}

The body weight was recorded at birth and on the 21st, 30th, 60th and 90th days of age with an appropriate balance 
(model AS-1000). The body length of the pups was recorded by the measurement of external surfaces (length from noseto-anus) with callipers $(0.01 \mathrm{~mm}$ accuracy). The body weights and body lengths of pups were used to determine the anthropometrical parameter of the Lee index (cube root of body weight $(\mathrm{g}) /$ nose-to-anus length $(\mathrm{cm}))^{(4)}$.

\section{Glucose and insulin tolerance test}

At 30 and $80 \mathrm{~d}$, the rats were fasted overnight for the OGTT and ITT tests. The OGTT was performed after administration of an oral glucose load $(2 \mathrm{~g} / \mathrm{kg})$ via gavage. Blood samples were taken from the tail veins before glucose administration and, subsequently, at 15, 30, 60 and $90 \mathrm{~min}$. At $24 \mathrm{~h}$ after OGTT, ITT was performed after an intraperitoneal injection of insulin ( $0.75 \mathrm{IU} / \mathrm{kg}$ body weight) and blood glucose concentrations were measured before $(0 \mathrm{~min})$ and after (30, 60, 90 and $120 \mathrm{~min}$ ). All measurements of blood glucose concentration were performed with an Accu-Check glucometer (Bayer)

\section{Biochemistry parameters and oxidative stress assay}

At 30 and $80 \mathrm{~d}$, offspring from both groups were anaesthetised with ketamine $(80 \mathrm{mg} / \mathrm{kg})$ and xylazine $(10 \mathrm{mg} / \mathrm{kg})$, and blood samples (approximately $2 \mathrm{ml}$ ) were collected by plexus retroorbital disruption and centrifuged at $300 \mathrm{~g}, 25^{\circ} \mathrm{C}$, during $15 \min ^{(4)}$. This experiment was performed $48 \mathrm{~h}$ after the glucose homoeostasis experiments. Serum samples were collected for the quantification of TC, HDL, VLDL, LDL, TAG and glucose with commercial kits. The concentration of malondialdehyde (MDA), an end product of lipid peroxidation, was measured as an indicative of oxidative stress. In this assay, MDA reacts with thiobarbituric acid to produce a red-coloured complex. Briefly, $400 \mu \mathrm{l}$ of perchloric acid ( $7 \%$ ) was added to $250 \mu \mathrm{l}$ of serum, mixed and centrifuged at $600 \mathrm{~g}, 4^{\circ} \mathrm{C}$, during $20 \mathrm{~min}$. The supernatant was collected, added to $400 \mu \mathrm{l}$ of thiobarbituric acid $(0.6 \%)$, heated at $100^{\circ} \mathrm{C}$ during $1 \mathrm{~h}$ and read at $532 \mathrm{~nm}$. A standard curve of MDA was constructed and the results were expressed as nmol of $\mathrm{MDA} / \mathrm{ml}^{(33)}$.

\section{Surgical procedure for blood pressure record in male rats at 90 - $d$-old}

At least $4 \mathrm{~d}$ after the plexus retro-orbital disruption experiments, BP measurements were performed as previously described ${ }^{(4)}$. For this experiment, eight rats used in each group come from four different litters. In brief, male (CTL, $n$ 8; DLP, $n$ 8) rats at $90 \mathrm{~d}$ old were anaesthetised with ketamine $(80 \mathrm{mg} / \mathrm{kg})$ and xylazine $(10 \mathrm{mg} / \mathrm{kg})$ to insert polyethylene catheters in the femoral artery and vein. The catheters were tunnelled through the back of the neck and ketoprofen $(5 \mathrm{mg} / \mathrm{kg})$ was injected subcutaneously. The offspring underwent a period of surgical recovery of $24 \mathrm{~h}$ until the $\mathrm{BP}$ record experiments began. Arterial pressure (AP) and heart rate (HR) were recorded in conscious animals connecting the arterial catheter to a pressure transducer (LabChart 7 Pro; ADInstruments).
Measurements and analysis of arterial blood pressure, ventilation and sympathetic tonus

The pulsatile AP was recorded for 40-50 min under basal conditions, and the values of the systolic arterial pressure (SAP), diastolic arterial pressure (DAP), mean arterial pressure (MAP) and HR were calculated by selection of $10 \mathrm{~min}$ for each animal. This same period was used for electrocardiogram (ECG) and spectral analysis.

After $40 \mathrm{~min}$ of cardiovascular recordings, measurements of the respiratory frequency $\left(\mathrm{R}_{\mathrm{F}}\right)$ were also performed by using the whole-body plethysmographic method ${ }^{(34)}$. Air flow was suspended for short periods $(3 \mathrm{~min})$, and breathing was captured by a pressure differential transducer connected to a signal amplifier (ML141 Spirometer; PowerLaband ADInstruments).

After recording the baseline ventilation measurements, the respiratory and cardiovascular responses to $\mathrm{CO}_{2}$ were induced by flushing a hypercapnic gas mixture $\left(7 \% \mathrm{CO}_{2}, 21 \% \mathrm{O}_{2}\right.$ and $\mathrm{N}_{2}$ balance; Linde Gas) into the plethysmographic chamber, as previously described by our research group ${ }^{(4)}$. $\mathrm{R}_{\mathrm{F}}$, tidal volume $\left(\mathrm{V}_{\mathrm{T}}\right)$ and ventilation minute $\left(\mathrm{V}_{\mathrm{E}}\right)$ were determined in an airconditioned room (control baseline) before and after the hypercapnic challenge. A period of $10 \mathrm{~s}$ was selected for the determination of mean $R_{F}$. $V_{T}$ was calculated using the formula described previously by Malan ${ }^{(34)}$ and $\mathrm{V}_{\mathrm{E}}$ acquired through product $\mathrm{R}_{\mathrm{F}} \times \mathrm{V}_{\mathrm{T}}$. The cardiovascular analysis was performed during the flow of the hypercapnic gas mixture. A period of $30 \mathrm{~s}$ of BP and HR was selected at 1, 2, 3, 4 and 5 min of gas flow. The cardiorespiratory reflex was assessed by the difference between the baseline and response observed after hypercapnia stimulus $(\Delta)$.

A period of 10 min after hypercapnic stimuli, a peripheral chemoreflex was activated through the intravenous injection of potassium cyanide (KCN; $40 \mathrm{mg} / 100 \mathrm{ml}$ per rat; Merck) in accordance with previous reports ${ }^{(26,30)}$. The difference between MAP, HR and $\mathrm{R}_{\mathrm{F}}$ baseline and the peak increased after the stimulus ( $\Delta$ MAP, $\Delta \mathrm{HR}$ and $\Delta \mathrm{R}_{\mathrm{F}}$ ) was assessed for, respectively, sympathetic, parasympathetic and respiratory components.

Last, $10 \mathrm{~min}$ after the peripheral chemoreflex activation, the contribution of the sympathetic vascular tone to cardiovascular system was evaluated by an intravenous injection of the ganglionar blocker (hexamethonium; $30 \mathrm{mg} / \mathrm{kg}$; Sigma-Aldrich ${ }^{\circledR}$ ). The sympathetic tonus was calculated by the difference between the MAP after the blocker and the baseline MAP.

All of the data were analysed offline with the use of appropriate software (LabChart 7 Pro; ADInstruments), and at the end of the research period the animals were euthanised with $0.5 \mathrm{ml}$ overdose of ketamine (endovenously). All protocols performed for the BP and respiratory parameter experiments are shown in Fig. 1.

\section{Electrocardiogram analysis}

ECG recordings were processed by computer software (ECG analysis module for LabChart Pro; ADInstruments) for automatic detection of the $\mathrm{R}$ waves and beat-by-beat calculation of $\mathrm{RR}$ interval. For detection of ECG, $12 \mathrm{~ms}$ was used to determine typical QRS width and for R waves at least $100 \mathrm{~ms}$ apart. For ECG analysis, $70 \mathrm{~ms}$ was used for the maximum PR and $90 \mathrm{~ms}$ for maximum RT. 
Maternal dyslipidaemia induces hypertension in male offspring

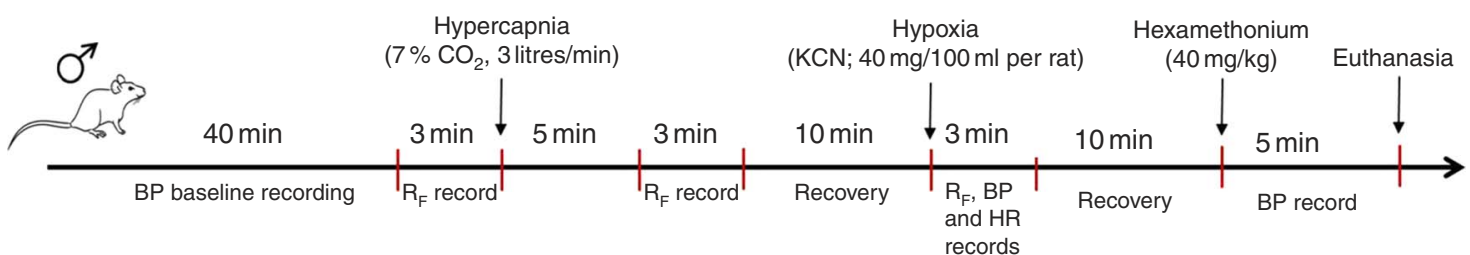

Fig. 1. Schematic protocol performed to record arterial blood pressure and ventilatory parameters. BP, blood pressure; $R_{F}$, respiratory frequency; HR, heart rate.

\section{Spectral analysis}

The cardiovascular autonomic evaluation was performed using the frequency domain analysis of the SAP and pulse interval (PI) by an appropriate software program (CardioSeries-v.2.4; www.danielpenteado.com). The spectra were integrated in the low-frequency (LF, 0.2-0.75 Hz) and the high-frequency (HF) bands $(0.75-3 \mathrm{~Hz})$. To assess the sympathovagal index, the LF: $\mathrm{HF}$ ratio of the variability was calculated. In addition, the spontaneous baroreflex sensitivity was calculated through a sequence method.

\section{Statistical analysis}

Each experimental group included at least one or two animals from each litter. Bartlett's test was performed to evaluate data homogeneity as to respiratory, cardiovascular and biochemical parameters, and statistical results supported the use of unpaired Student's $t$ tests. Two-way ANOVA and Bonferroni's post hoc test were used for analysis of food intake, body weight, OGTT and ITT during pregnancy and lactation. Statistical analysis was conducted with GraphPad Prism 5 program for Windows (GraphPad Software ${ }^{\circledR}$, Inc.). The values are presented as means with their standard errors. Values of $P<0.05$ were considered as statistically significant.

\section{Results}

Food intake, body weight and metabolic profile in dams exposed to a dyslipidaemic diet during pregnancy and lactation

As observed in Fig. 2, after weaning, DLP dams presented significantly raised serum levels of TC (4.0-fold), TAG (2.0-fold), VLDL + LDL (7·7-fold) and reduced HDL-cholesterol (2.4-fold) when compared with CTL dams. We also observed that the atherogenic indexes TAG/HDL, TC/HDL and VLDL $+\mathrm{LDL} / \mathrm{HDL}$ were all increased in the DLP dams $(P<0 \cdot 05$, Fig. 2).

After weaning, the DLP dams exhibited higher hepatic lipid accumulation than the CTL dams (Fig. 2). We measured the food intake and body weight during pregnancy and lactation. Despite the reduced food intake in the last 2 weeks of lactation, there were no differences in the body weight and total weight gain between the CTL and DLP dams (online Supplementary Fig. S1).

In order to evaluate whether the DLP diet during pregnancy and lactation would alter the regulation of glucose homoeostasis, we conducted OGTT and ITT at the end of lactation.
As shown in Fig. 3, the DLP dams exhibited higher serum glucose in 15 (9.7 (SEM 1.7) v. $6.2($ SEM 0.4) mmol/l, $P<0.05)$ and $30 \mathrm{~min}(9.2(\operatorname{sem~} 0.8) v .6 .3(\mathrm{sem} 0.5) \mathrm{mmol} / \mathrm{l}, P<0.05)$ of OGTT when compared with CTL. In agreement, the DLP dams presented greater AUC $90 \mathrm{~min}$ after OGTT $(P<0 \cdot 05$, Fig. 3). Similarly, the DLP dams also exhibited higher serum glucose after intraperitoneal insulin injection in 30 (3.4 (SEM 0.16) v. 2.2 (SEM $0.21) \mathrm{mmol} / \mathrm{l}, P<0.05)$ and $120 \mathrm{~min}(3.12$ (SEM 0.56) v. 1.5 (SEM $0 \cdot 19) \mathrm{mmol} / \mathrm{l}, P<0.05)$ and consequently greater AUC $120 \mathrm{~min}$ after the injection of insulin $(P<0 \cdot 05$, Fig. 3$)$.

Together, these results show that excessive consumption of saturated trans-fatty acids and cholesterol during pregnancy and lactation induce a maternal dysmetabolic condition characterised by dyslipidaemia and altered glucose tolerance and insulin sensitivity.

\section{Short-term effect of maternal dyslipidaemia on somatic growth and biochemical parameters in juvenile male offspring}

Fig. 4 shows the somatic growth curves from birth to 90 days. Body weight in the rats exposed to maternal dyslipidaemia was lower at birth (5.2 (sem 0.08) v. 5.6 (sem 0.1) g, $P<0.05$, Fig. 4) and weaning (39.5 (sem 0.7) v. 44.5 (sem 0.6) g, $P<0.05$, Fig. 4) when compared with the CTL rats. From weaning to $60 \mathrm{~d}$, the body weight was similar between groups. However, at $90 \mathrm{~d}$, the DLP rats exhibited significantly higher body weight (362 (SEM 4.7) v. 336 (SEM 4.9) g, $P<0.05$, Fig. 4) in comparison with the CTL rats. Despite the changes in body weight, no change was observed in body length $(P>0.05 ;$ Fig. 4$)$ and Lee index (P>0.05; Fig. 4).

Regarding the short-term effects of maternal dyslipidaemia on glucose handling in male offspring ( $30 \mathrm{~d}$ after birth), despite the fact that the DLP offspring exhibited increased glucose at $15 \mathrm{~min}$ (4.3 (sem 0.3) v. 3.5 (sem 0.19) $\mathrm{mmol} / \mathrm{l}, P<0.05$, Fig. 5) and a tendency for increased AUC of OGTT $(P=0 \cdot 06$; Fig. 5$)$, the fasting serum glucose concentration was similar between groups ( $P>0.05$, Fig. 5 ). Serum lipid profiles of the male offspring reveal that serum TC and VLDL cholesterol concentrations were increased in DLP rats $(P<0.05 ;$ Fig. 5$)$. The TAG and HDL-cholesterol concentrations were not different between CTL and DLP offspring (Fig. 5).

\section{Long-term effect of maternal dyslipidaemia on the biochemical and cardiorespiratory parameters in male rat offspring}

Investigating the long-term effects of maternal dyslipidaemia on glucose handling ( $80 \mathrm{~d}$ of age), insulin sensitivity and the serum 
(a)

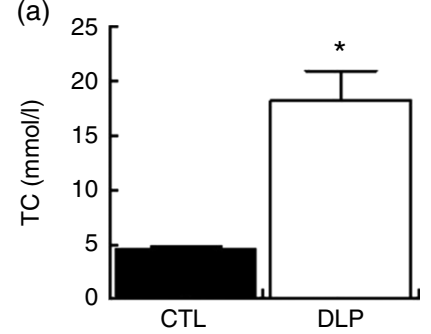

(d)

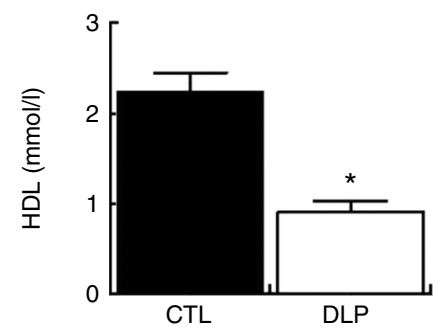

(b)

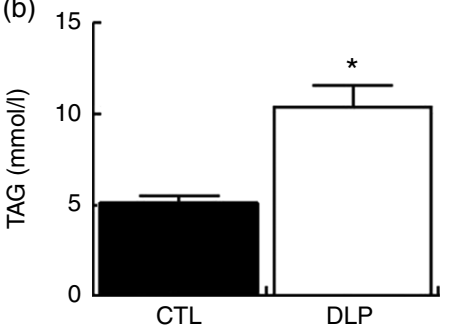

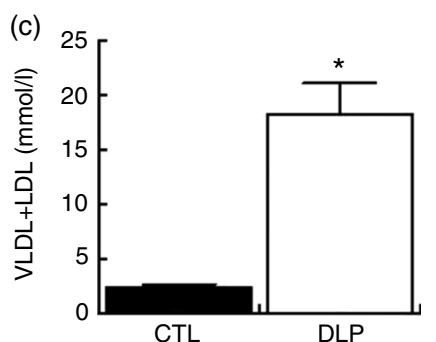

(e)

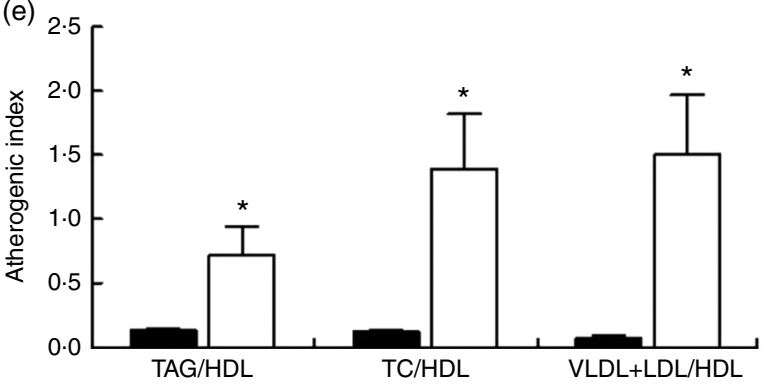

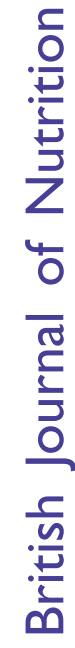

(f)

CTL

DLP

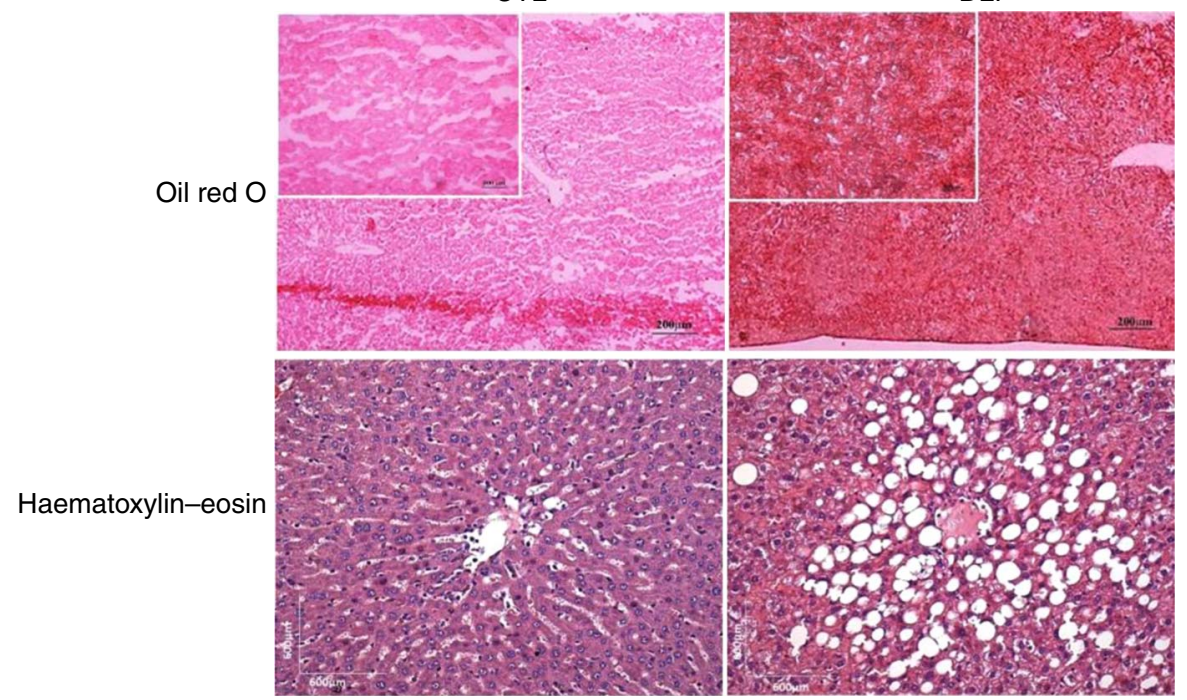

Fig. 2. Fasting serum total cholesterol (TC) (a), TAG (b), VLDL + LDL (c) and HDL (d), atherogenic index (e), hepatic (f) and intestine (g) lipid accumulation in dams that received a control (CTL) or dyslipidaemic (DLP) diet during pregnancy and lactation. Groups: CTL group $(n 5)$ and DLP group. Values are means with their standard errors represented by vertical bars. ${ }^{*} P<0.05 \mathrm{v}$. CTL using unpaired Student's $t$ test.

(a)

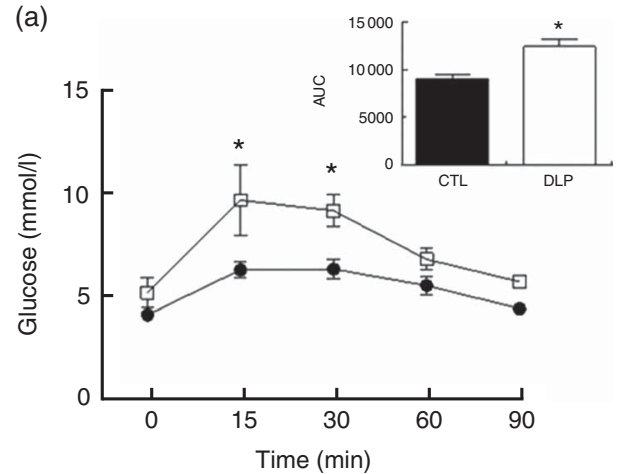

(b)

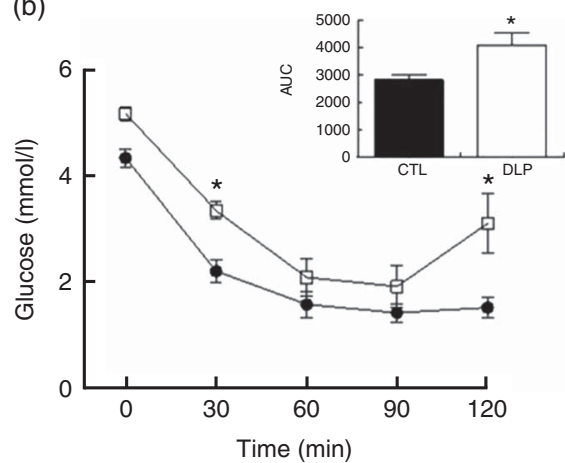

Fig. 3. Oral glucose tolerance test (a) and insulin tolerance test (b) in dams that received a control (CTL) or dyslipidaemic (DLP) diet during pregnancy and lactation. Groups: CTL group $(n 5,--)$ and DLP group $(n 5,-\square)$. Values are means with their standard errors represented by vertical bars. ${ }^{*} P<0.05 \mathrm{v}$. CTL using two-way ANOVA, followed by Bonferroni's post hoc test or Student's $t$ test. 
(a)

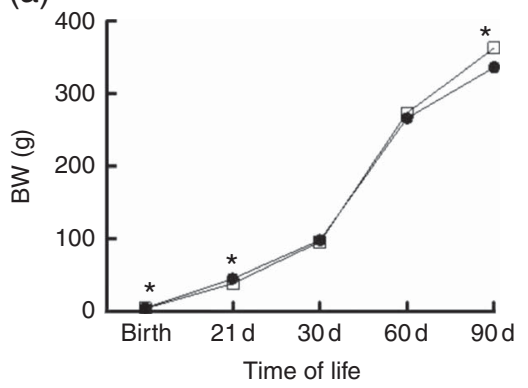

(b)

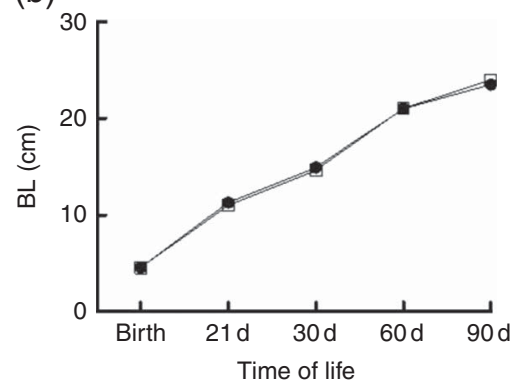

(c)

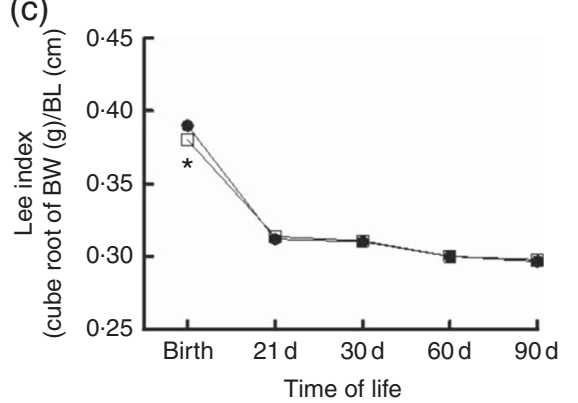

Fig. 4. Body weight (BW) (a), body length (BL) (b) and Lee index (c) in male offspring from dams exposed to maternal dyslipidaemia during pregnancy and lactation. Groups: control group (CTL, $n$ 20, - - ) and dyslipidaemic group (DLP, $n$ 20, $\square$ ). Values are means with their standard errors represented by vertical bars. ${ }^{\star} P<0.05 v$. CTL using two-way ANOVA, followed by Bonferroni's post hoc test or Student's $t$ test.
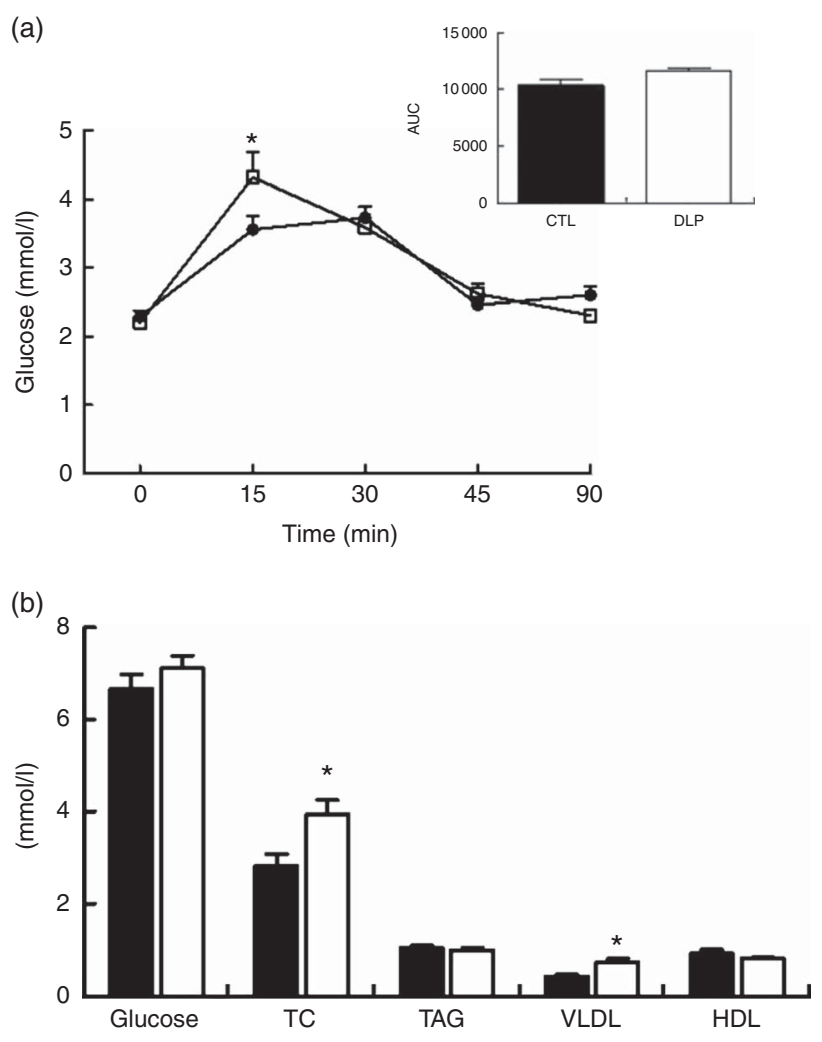

Fig. 5. Oral glucose tolerance test (a) and fasting serum of glucose, total cholesterol (TC), TAG, VLDL and HDL (b) in male offspring at $30 \mathrm{~d}$ old from dams exposed to maternal dyslipidaemia during pregnancy and lactation. Groups: control group (CTL, $n$ 8) and dyslipidaemic group (DLP, $n$ 8). Values are means with their standard errors represented by vertical bars. a: - , CTL; $\square$, DLP; b: $\square, C T L ; \square$, DLP. * $P<0.05$ v. CTL using twoway ANOVA, followed by Bonferroni's post hoc test or Student's $t$ test.

lipid profile, no difference was observed between CTL and DLP male offspring ( $P>0 \cdot 05$; Fig. 6).

On examining male offspring at $90 \mathrm{~d}$, we found that the DLP rats displayed an increase in SAP, DAP and MAP in comparison with the CTL group $(P<0 \cdot 05$; Table 2$)$. The arterial hypertension observed in our model was not associated with tachycardia, as evidenced by the similar baseline HR between the CTL and DLP groups ( $P>0 \cdot 05$, Table 2$)$. Although BP and HR are interrelated components of the cardiovascular system, resting $\mathrm{HR}$ and $\mathrm{BP}$ do not necessarily increase at the same rate in hypertensive condition. The inverse proportion between BP and HR may be due to increased stiffness of the large arteries, such as the aorta and large elastic arteries. Spectral analysis using baseline BP recordings demonstrated that the LF range of SAP and the LF:HF ratio of the PI were augmented in the DLP group $(P<0 \cdot 05$; Table 2$)$. In relation to spontaneous baroreflex sensitivity, both the CTL and DLP rats exhibited similar baroreflex gain $(P>0.05$, Table 2$)$. In addition, all ECG measures analysed, such as RR, PR, QRS and QT intervals and P duration, were not different between the CTL and DLP groups $(P>0.05$, Table 2). Representative baseline recordings of pulsatile arterial pressure (PAP), MAP and HR in male rat offspring are shown in the online Supplementary Fig. S2.

Under the baseline condition, $\mathrm{R}_{\mathrm{F}}, \mathrm{V}_{\mathrm{T}}$ and $\mathrm{V}_{\mathrm{E}}$ values were similar between the CTL and DLP male offspring $(P>0.05$; Fig. 7). Hypercapnia $\left(\mathrm{CO}_{2} 7 \%\right)$ produced an increase in $\mathrm{R}_{\mathrm{F}}$, $\mathrm{V}_{\mathrm{T}}$ and $\mathrm{V}_{\mathrm{E}}$ values in both the CTL and DLP groups (online Supplementary Fig. S2). In comparison with CTL offspring, the DLP rats exhibited higher values of $\Delta \mathrm{R}_{\mathrm{F}}$ (Fig. 6) and $\Delta \mathrm{V}_{\mathrm{E}}$ (Fig. 7), but no difference in $\Delta V_{T}$ (Fig. 7) values after hypercapnia. Breathing recordings of representative rats from the CTL and DLP groups during the basal and hypercapnic conditions are shown in the online Supplementary Fig. S3. In addition, during the $5 \mathrm{~min}$ of hypercapnic gas flushing, the MAP (Fig. 7) was augmented in the DLP group when compared with the CTL group, but no difference in HR was observed (Fig. 7).

Peripheral chemoreflex activation produced pressor, bradycardic and tachypnoeic responses in both groups (Fig. 8). The increases in MAP $(P<0.05$; Fig. 8$)$ and $\mathrm{R}_{\mathrm{F}}(P<0.05$; Fig. 8) were significantly greater in the DLP group than in the CTL group. In contrast, the magnitude of the decrease in HR showed a tendency towards reduction in the DLP rats ( $P=0.06$, Fig. 8). Together, these results suggest that the DLP offspring exhibited increased sensitisation of cardiorespiratory chemoreceptors.

To test whether sympathetic tonus was augmented in DLP rats at 90 days, we evaluated the changes in MAP ( $\triangle \mathrm{MAP}$ ) after administration of a pharmacological ganglionic blocker in both groups. After the blockage, the DLP rats showed increased $\triangle$ MAP $(P<0.05$; Fig. 9) when compared with CTL rats. 
(a)

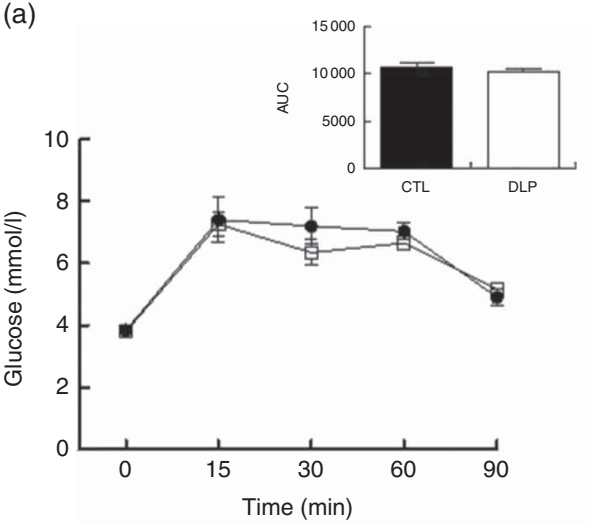

(b)

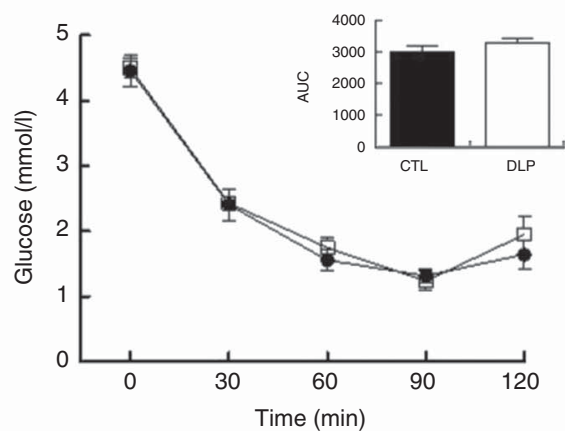

(c)

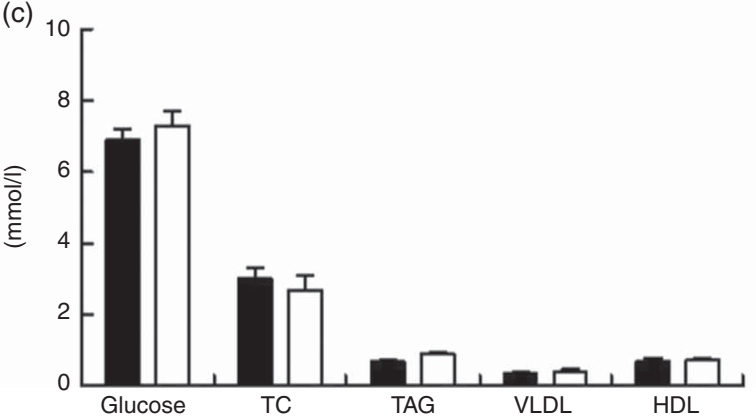

Fig. 6. Oral glucose tolerance test (a), insulin tolerance test (b) and fasting serum of glucose, total cholesterol (TC), TAG, VLDL and HDL (c) in male offspring at $80 \mathrm{~d}$ old from dams exposed to maternal dyslipidaemia during pregnancy and lactation. Groups: control (CTL, $n$ 8) and dyslipidaemic group (DLP, $n$ 8). Values are means with their standard errors represented by vertical bars. a and b: - - CTL; $\square$, DLP; $c$ : $\square$, CTL; $\square$, DLP. * $P<0.05$ v. CTL using two-way ANOVA, followed by Bonferroni's post hoc test or Student's $t$ test.

Table 2. Baseline parameters of blood pressure in male offspring at $90 \mathrm{~d}$ old from control and dyslipidaemic dams (Mean values with their standard errors)

\begin{tabular}{|c|c|c|c|c|}
\hline \multicolumn{5}{|c|}{ Male offspring } \\
\hline & \multicolumn{2}{|c|}{ CTL group } & \multicolumn{2}{|c|}{ DLP group } \\
\hline & Mean & SEM & Mean & SEM \\
\hline \multicolumn{5}{|c|}{ Cardiovascular parameters } \\
\hline SAP $(\mathrm{mmHg})$ & 127 & $7 \cdot 3$ & $155^{\star}$ & $5 \cdot 0$ \\
\hline $\mathrm{DAP}(\mathrm{mmHg})$ & 74 & $7 \cdot 0$ & $103^{*}$ & 1.8 \\
\hline MAP $(\mathrm{mmHg})$ & 90 & 5.5 & $121^{*}$ & $2 \cdot 9$ \\
\hline $\mathrm{HR}$ (bpm) & 361 & 14 & 346 & 16 \\
\hline \multicolumn{5}{|l|}{ Spectral analysis } \\
\hline $\mathrm{LF}\left(\mathrm{mmHg}^{2}\right)$ & 4.8 & 0.4 & $9.0^{*}$ & 0.9 \\
\hline $\mathrm{HF}\left(\mathrm{mmHg}^{2}\right)$ & 5.9 & 1.3 & 3.0 & 0.4 \\
\hline LF:HF & 0.13 & 0.02 & $0.29^{*}$ & 0.007 \\
\hline $\mathrm{BRS}(\mathrm{ms} / \mathrm{mmHg})$ & $1 \cdot 18$ & 0.14 & $1 \cdot 21$ & $0 \cdot 10$ \\
\hline \multicolumn{5}{|l|}{ ECG analysis } \\
\hline RR interval (s) & 0.165 & 0.003 & $0 \cdot 171$ & 0.014 \\
\hline PR interval (s) & 0.049 & 0.002 & 0.047 & 0.001 \\
\hline $\mathrm{P}$ duration (s) & 0.031 & 0.002 & 0.028 & 0.001 \\
\hline QRS interval (s) & 0.033 & 0.002 & 0.034 & 0.002 \\
\hline QT interval (s) & 0.085 & 0.004 & 0.083 & 0.006 \\
\hline
\end{tabular}

SAP, systolic arterial pressure; DAP, diastolic arterial pressure; MAP, mean arterial pressure; HR, heart rate; bpm, beats per min; LF, low-frequency band; HF, highfrequency band; BRS, spontaneous baroreflex sensitivity; ECG, electrocardiogram. * $P<0.05$.

Short- and long-term effects of maternal dyslipidaemia on oxidative stress in male offspring

The quantification of serum MDA is summarised in Fig 10. MDA concentrations in DLP rats were increased at $30 \mathrm{~d}(P<0 \cdot 05$; Fig. 10) and $90 \mathrm{~d}$ of age $(P<0.05$; Fig. 10) when compared with the CTL rats.

\section{Discussion}

Previous studies have reported that high dietary fat, cholesterol and sugar intake during pregnancy ${ }^{(14,35)}$ or lactation ${ }^{(36)}$ impair lipid and glucose metabolism in mouse and rat dams.

To the best of our knowledge, here we have demonstrated for the first time that dams fed a DLP diet during pregnancy and lactation periods exhibit high levels of cholesterol, TAG, atherogenic index, compromised glucose tolerance, insulin sensitivity and hepatic steatosis at weaning. These findings suggest that an excessive consumption of a diet rich in saturated trans-fatty acids and cholesterol during pregnancy and lactation may be a useful experimental model for studying the effects of dyslipidaemia in dams and in their offspring.

Similarly to previous studies, we have observed that pups from dams fed a DLP diet had low weight at birth and weaning, but recovered normal body weight at $30 \mathrm{~d}$ of age ${ }^{(14,35)}$. Interestingly, a recent meta-analysis of clinical trials found that maternal dyslipidaemia during pregnancy was associated with an increased risk of preterm birth ${ }^{(10)}$. Together, this evidence reveals that high levels of maternal lipid may increase the risk of low birth weight.

In adult life, DLP rats exhibited higher body weight in comparison with the CTL offspring. Both groups, however, presented a similar Lee index. Body fat composition was not analysed, so it is not possible to state that DLP offspring displayed obesity. Future studies investigating possible development of obesity will be focused in our next research. 

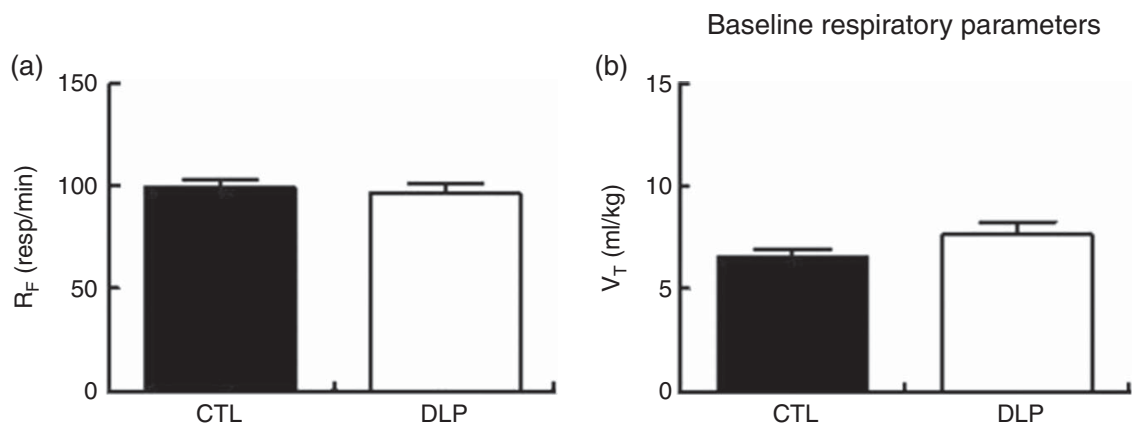

$\mathrm{CO}_{2}$ chemosensitivity

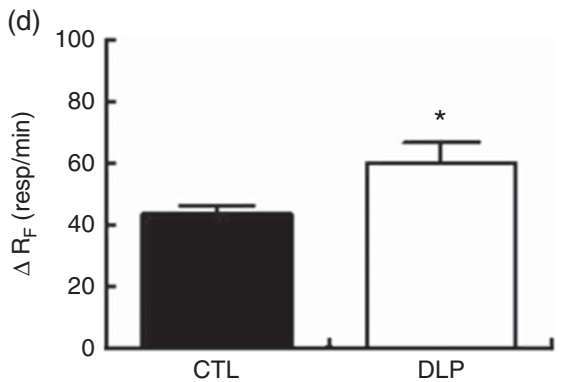

(e)

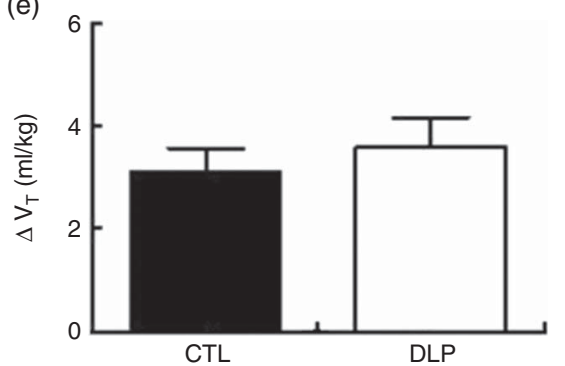

(h)

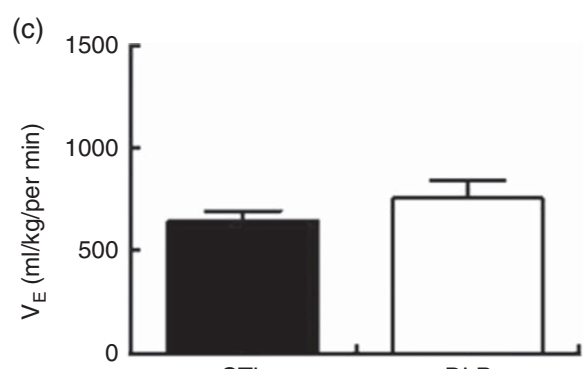

CTL

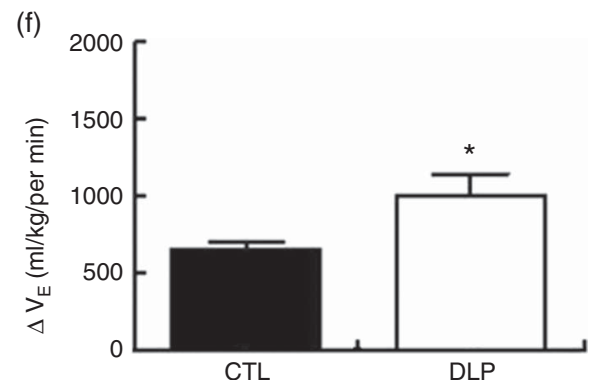

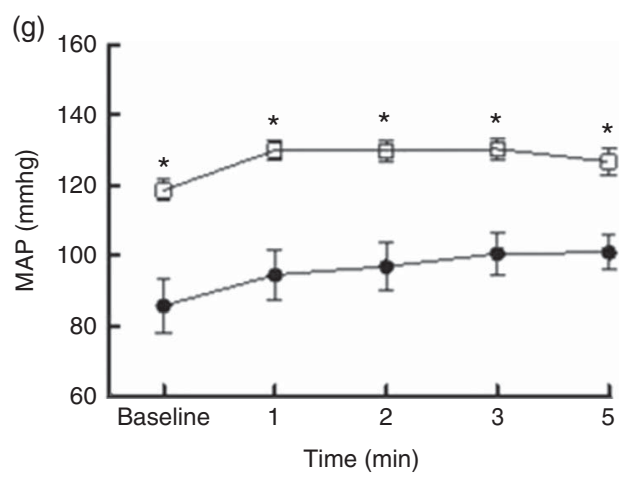

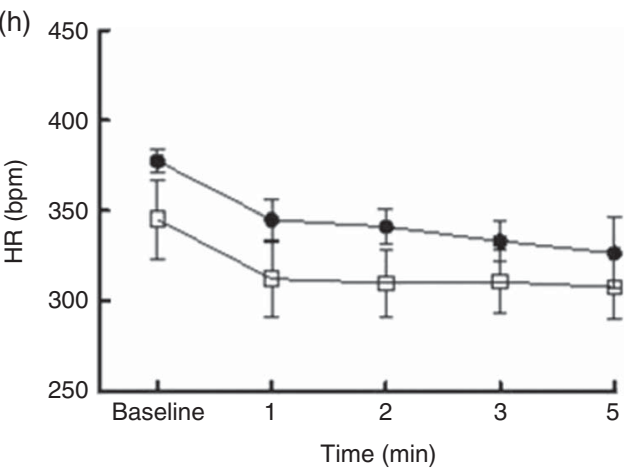

Fig. 7. Respiratory frequency $\left(\mathrm{R}_{\mathrm{F}}\right)$, tidal volume $\left(\mathrm{V}_{\mathrm{T}}\right)$ and ventilation minute $\left(\mathrm{V}_{\mathrm{E}}\right)$ at rest $(\mathrm{a}-\mathrm{c})$ and after hypercapnia $\left(7 \% \mathrm{CO}_{2}\right)(\mathrm{d}-\mathrm{f})$. During the 5 min of hypercapnia, the values of mean arterial pressure (MAP, g) and heart rate $(\mathrm{HR}, \mathrm{h})$ were recorded in male offspring at $90 \mathrm{~d}$ old from dams exposed to maternal dyslipidaemia during pregnancy and lactation. Groups: control group (CTL, $n 10)$ and dyslipidaemic group (DLP, $n$ 10). Values are means with their standard errors represented by vertical bars. g and $\mathrm{h}:-1, \mathrm{CTL} ; \square, \mathrm{DLP} .{ }^{*} P<0.05 \mathrm{v}$. CTL using two-way ANOVA, followed by Bonferroni's post hoc test or Student's $t$ test. bpm, Beats per min.

In our study, serum TC and LDL-cholesterol were maintained at a high level in DLP offspring at $30 \mathrm{~d}$ of age. It has been shown that impaired glucose tolerance associated with high serum levels of cholesterol, TAG and fatty liver are observed in pups from dams fed a high-fat or high-cholesterol diet during pregnancy or lactation $^{(36,37)}$. These alterations possibly occur as a consequence of high cholesterol and fat content in the dams' milk ${ }^{(36)}$.

In agreement with other studies that used maternal high-fat diet as a model ${ }^{(9)}$, our study showed that male offspring exposed to maternal dyslipidaemia during pregnancy and lactation developed arterial hypertension at $90 \mathrm{~d}$ of age. It should be noted, however, that another study reported that offspring from dams exposed to hypercholesterolaemia during pregnancy did not exhibit hypertension in adulthood ${ }^{(38)}$. However, in that study, BP was measured in anaesthetised rats, limiting the comparison with our results.

It is well accepted that the autonomic dysfunction, verified by excessive sympathetic tone and vagal withdrawal, alters the vascular reactivity and contributes to the development of hypertension in both hypertensive experimental models and hypertensive patients ${ }^{(27,30,39,40)}$.

The LF and HF ranges or the LF:HF ratio represent more accurately the sympathetic activity and autonomic modulation $^{(41)}$. In addition to these methods for estimating sympathetic activity, we used an experimental protocol involving a ganglionic sympathetic blockade with hexamethonium. We observed in our study that LF power, LF:HF ratio and delta for arterial pressure variation ( $\triangle \mathrm{MAP}$ ) after hexamethonium infusion were increased in the DLP offspring. Together, our results support the hypothesis that the arterial hypertension observed in male rats from dams exposed to dyslipidaemia during pregnancy and lactation is associated with sympathetic hyperactivity. It is interesting to note that increased LF power, LF:HF index and great delta arterial pressure variation after hexamethonium infusion are observed in hypertensive rats ${ }^{(41)}$ and onset hypertension in men ${ }^{(42)}$. 
(a)
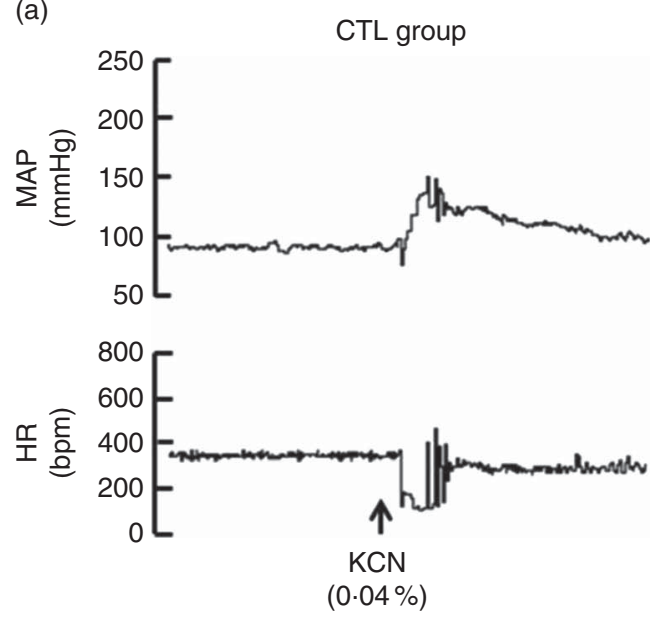
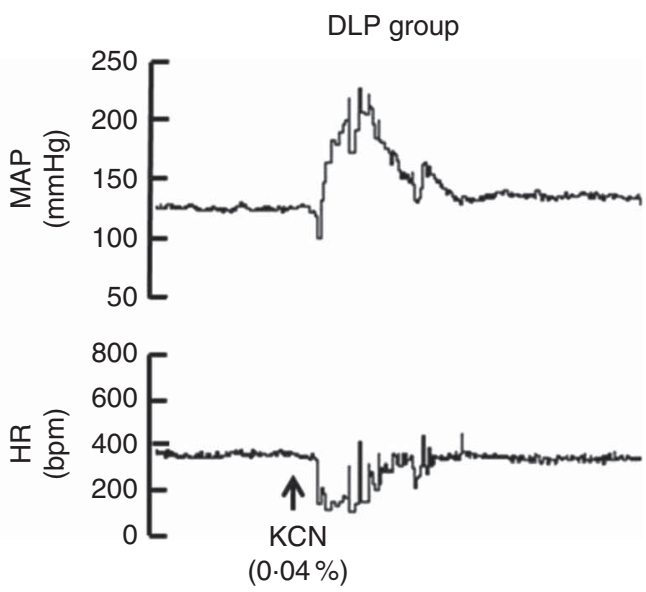

(b)

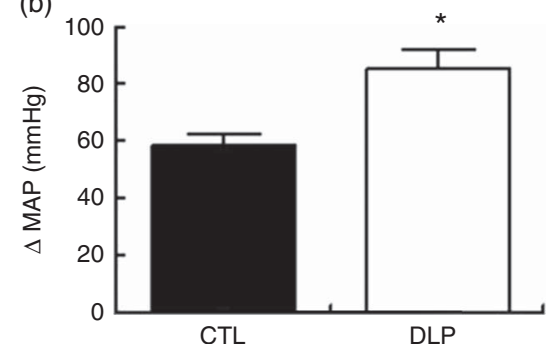

(c)

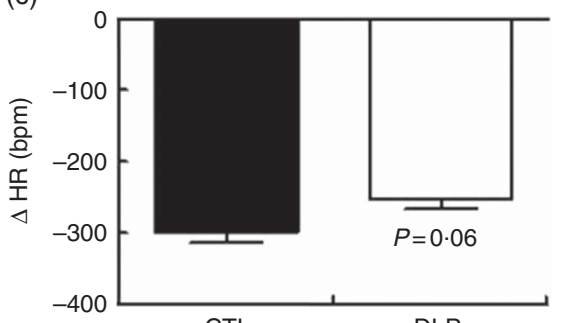

(d)

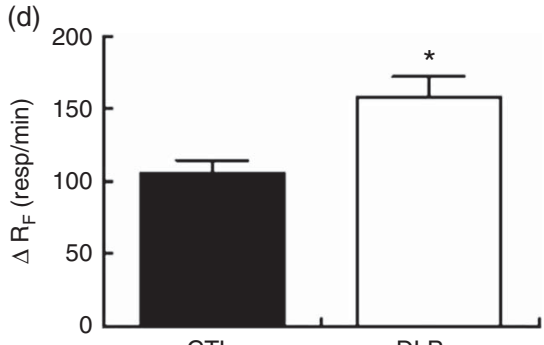

Fig. 8. Representative recording of mean arterial pressure (MAP) and heart rate (HR) during chemoreflex activation (a) and evaluation of variation in MAP (b), HR (c) and respiratory frequency (d) during chemoreflex activation ( $\mathrm{KCN}, 0.04 \%)$ in male offspring at $90 \mathrm{~d}$ old from dams exposed to maternal dyslipidaemia during pregnancy and lactation. Groups: control group (CTL, $n 10)$ and dyslipidaemic group (DLP, $n$ 10). Values are means with their standard errors represented by vertical bars. ${ }^{*} P<0.05$ v. CTL using Student's $t$ test. bpm, Beats per min.

(a)
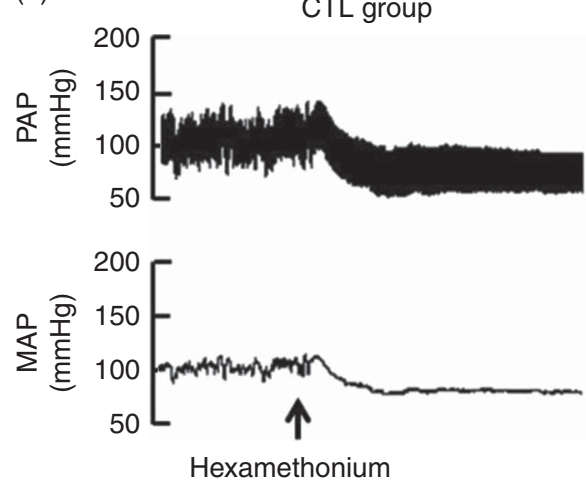

$(30 \mathrm{mg} / \mathrm{kg})$
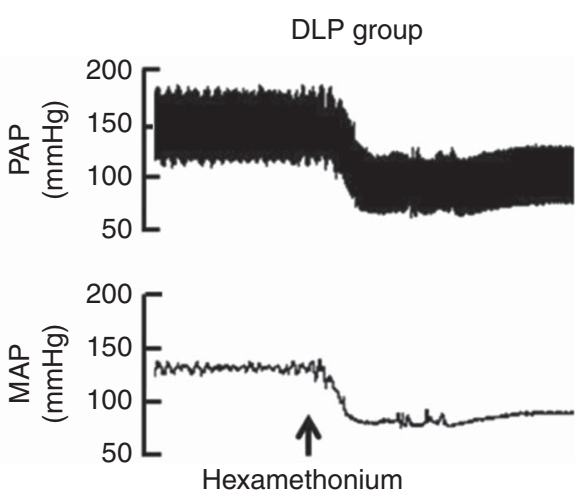

$(30 \mathrm{mg} / \mathrm{kg})$ (b)

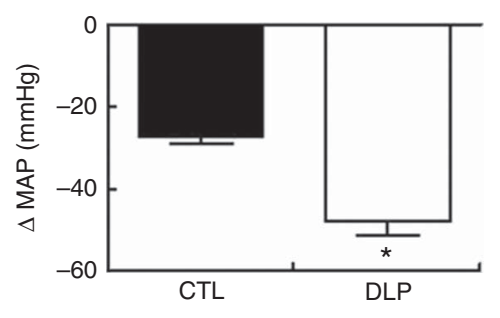

Fig. 9. Representative tracings of pulsatile arterial pressure (PAP) and mean arterial pressure (MAP) after hexamethonium (a) and evaluation of delta change of the MAP (b) in male offspring at $90 \mathrm{~d}$ old from dams exposed to maternal dyslipidaemia during pregnancy and lactation. Groups: control group (CTL, $n$ 10) and dyslipidaemic group (DLP, $n$ 10). Values are means with their standard errors represented by vertical bars. ${ }^{\star} P<0.05 v$. CTL using Student's $t$ test.

It has been reported that a baroreflex dysfunction could lead to a sympathetic over-activity and subsequent development of hypertension $^{(43)}$. In addition, sympathetic outflow and BP have been shown to be modulated by the rhythmicity of the respiratory nervous system ${ }^{(44)}$. Part of this modulatory effect is influenced by the peripheral and central respiratory chemoreceptors. Activation of these chemoreceptors produces a powerful activation of the cardiorespiratory neuronal network and enhances the sympathetic and respiratory outflow ${ }^{(45)}$.
On analysing spontaneous baroreflex sensitivity, we found no differences between the groups, suggesting that baroreflex control, at least within the narrow range of the physiological variations of $\mathrm{BP}$, is not altered in adult rats exposed to maternal dyslipidaemia. This result differed from another study showing a reduction in the baroreflex control in male offspring from dams fed a westernised $\operatorname{diet}^{(9)}$.

The critical window relevant for the control of breathing and chemosensory consists of that period when respiratory control 
(a)

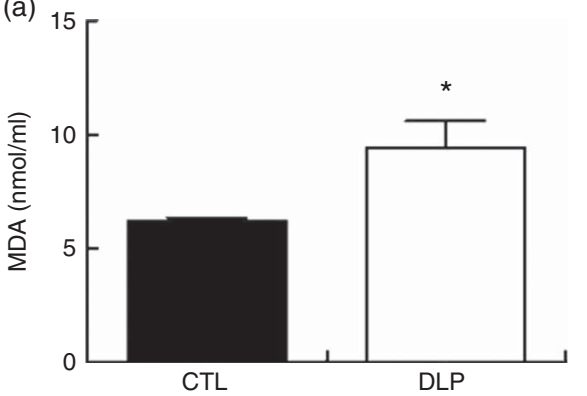

(b)

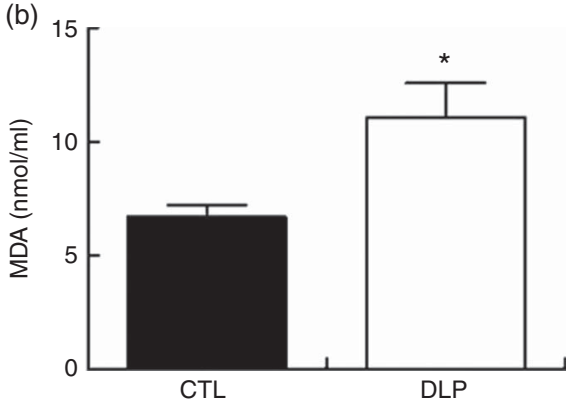

Fig. 10. Serum malondialdehyde (MDA) concentration at $30 \mathrm{~d}$ of age (a) and $80 \mathrm{~d}$ old (b) in male offspring from dams exposed to maternal dyslipidaemia during pregnancy and lactation. Groups: control group (CTL, $n$ 8) and dyslipidaemic group (DLP, $n$ 8). Values are means with their standard errors represented by vertical bars. ${ }^{*} P<0.05$ v. CTL using Student's $t$ test.

is still too immature and incapable of mounting an effective defence against acute respiratory challenges ${ }^{(46)}$. A number of environmental factors such as stress stimuli or oxygen levels experienced during early life can elicit, in the short or long term, developmental plasticity in the respiratory control system, which may be exemplified through changes in pulmonary ventilation or the hypoxic/hypercapinc ventilatory response $e^{(46,47)}$.

Our research group has convincingly demonstrated that nutritional insults during pregnancy and lactation can have an impact on the development of the respiratory phenotype, such as causing changes in baseline ventilation and hypoxic and hypercapnic ventilatory response ${ }^{(4)}$. Here, we have demonstrated that cardiovascular and ventilatory reflex responses to hypercapnia and hypoxia intensified significantly in male rats exposed to dyslipidaemia during pregnancy and lactation.

Clinical and experimental studies have shown that high levels of inflammatory markers (e.g. IL-6 and TNF) associated with high reactive $\mathrm{O}_{2}$ species (ROS) are found in the plasma and placenta from pregnant women with metabolic disorders or obesity $^{(48)}$. Together, pro-inflammatory cytokine and ROS could impair placental functioning and induce adverse effects on the fetus $^{(13,49)}$.

A recent study showed that inflammation-induced discharge of PGE2 alters breathing patterns and increases ventilatory response to $\mathrm{CO}_{2}{ }^{(50)}$. Interestingly, Samarasinghe and colleagues demonstrated that the ventilatory responses to hypoxia and hypercapnia were intensified in mouse offspring exposed to prenatal inflammation in the final days of gestation ${ }^{(51)}$. It was also shown that oxidative stress augments chemoreflex sensitivity in rats exposed to chronic intermittent hypoxia ${ }^{(17)}$. Our finding was that MDA levels, a biomarker used to assess oxidative stress, were increased in the serum of DLP offspring.

Considering the complementary approaches used in the present study, we propose, on the basis of our results, that environmental stress caused by maternal dyslipidaemia increases chemoreceptor sensitivity, which is a primary contribution to sympathetic hyperactivity. Then, these changes may reinforce the development of hypertension in maternal dyslipidaemia-induced hypertension model. It is, however, important to note that we investigated only male offspring. A growing number of studies have related that onset of hypertension evidenced in offspring from maternal nutritional insults dams is sex-dependent. The findings related that female offspring exposed to a nutritional insult during fetal life can be resistant to the development of hypertension in young adulthood $^{(48,52)}$. Our study was limited by the lack of pro-inflammatory cytokine and stress oxidative marker measurements in dams and of investigation of the molecular mechanisms associated with our physiological findings. These gaps will be investigated in future studies.

In conclusion, the present study shows that male offspring from DLP dams exhibit hypertension in adult life combined with sympathetic hyperactivity and amplified ventilatory and autonomic responses to hypoxia and hypercapnia.

\section{Acknowledgements}

The English text of this paper has been revised by Sidney Pratt, Canadian, MAT (The Johns Hopkins University), RSAdip - TESL (Cambridge University).

V. A. B. and M. M. are recipients of the CNPq Fellowship.

K. S. L. G., E. V. d. A. and J. L. d. B. A. designed the research and conducted the experiments; K. S. L. G., E. V. d. A., J. S. A., C. M. B., D. A. G., J. H. C.-S., M. M., H. V., V. A. B. and J. L. d. B. A. analysed the data and performed the statistical analysis; and K. S. L. G., V. A. B., H. V. and J. L. d. B. A. had primary responsibility for the final content. All authors read and approved the final manuscript.

The authors declare that there are no conflicts of interest.

\section{Supplementary material}

For supplementary material/s referred to in this article, please visit https://doi.org/10.1017/S0007114517003014

\section{References}

1. Landsberg L, Aronne LJ, Beilin LJ, et al. (2013) Obesity-related hypertension: pathogenesis, cardiovascular risk, and treatment: a position paper of The Obesity Society and the American Society of Hypertension. J Clin Hypertens (Greenwich) 15, 14-33.

2. Mills KT, Bundy JD, Kelly TN, et al. (2016) Global disparities of hypertension prevalence and control: a systematic analysis of population-based studies from 90 countries. Circulation. 134, 441-450. 
3. Moreira AP, Texeira TF, Ferreira AB, et al. (2012) Influence of a high-fat diet on gut microbiota, intestinal permeability and metabolic endotoxaemia. Br J Nutr 108, 801-809.

4. de Brito Alves JL, Nogueira VO, de Oliveira GB, et al. (2014) Short- and long-term effects of a maternal low-protein diet on ventilation, $\mathrm{O}(2) / \mathrm{CO}(2)$ chemoreception and arterial blood pressure in male rat offspring. Br J Nutr 111, 606-615.

5. Tennant IA, Barnett AT, Thompson DS, et al. (2014) Impaired cardiovascular structure and function in adult survivors of severe acute malnutrition. Hypertension 64, 664-671.

6. Chandi A, Sirohiwal D \& Malik R (2015) Association of early maternal hypertriglyceridemia with pregnancy-induced hypertension. Arch Gynecol Obstet 292, 1135-1143.

7. Costa-Silva JH, Simoes-Alves AC \& Fernandes MP (2016) Developmental origins of cardiometabolic diseases: role of the maternal diet. Front Physiol 7, 504.

8. Longo M, Refuerzo JS, Mann L, et al. (2016) Adverse effect of high-fat diet on metabolic programming in offspring born to a murine model of maternal hypertension. Am J Hypertens 29, 1366-1373.

9. Vidal-Santos R, Macedo FN, Santana MN, et al. (2017) Western diet in the perinatal period promotes dysautonomia in the offspring of adult rats. J Dev Orig Health Dis $\mathbf{8}$, 216-225.

10. Jiang S, Jiang J, Xu H, et al. (2017) Maternal dyslipidemia during pregnancy may increase the risk of preterm birth: a meta-analysis. Taiwan J Obstet Gynecol 56, 9-15.

11. Palinski W, Nicolaides E, Liguori A, et al. (2009) Influence of maternal dysmetabolic conditions during pregnancy on cardiovascular disease. $J$ cardiovasc Transl Res $\mathbf{2}$, 277-285.

12. Loy SL, Kns S \& Jm HJ (2013) Increase in maternal adiposity and poor lipid profile is associated with oxidative stress markers during pregnancy. Prev Med 57, Suppl., S41-S44.

13. Roberts KA, Riley SC, Reynolds RM, et al. (2011) Placental structure and inflammation in pregnancies associated with obesity. Placenta 32, 247-254.

14. Musial B, Vaughan OR, Fernandez-Twinn DS, et al. (2017) A Western-style obesogenic diet alters maternal metabolic physiology with consequences for fetal nutrient acquisition in mice. J Physiol 595, 4875-4892.

15. Monteiro MM, Franca-Silva MS, Alves NF, et al. (2012) Quercetin improves baroreflex sensitivity in spontaneously hypertensive rats. Molecules 17, 12997-13008.

16. Del Rio R, Moya EA, Parga MJ, et al. (2012) Carotid body inflammation and cardiorespiratory alterations in intermittent hypoxia. Eur Respir J 39, 1492-1500.

17. Morgan BJ, Bates ML, Rio RD, et al. (2016) Oxidative stress augments chemoreflex sensitivity in rats exposed to chronic intermittent hypoxia. Respir Physiol Neurobiol 234, 47-59.

18. Nunes FC, Ribeiro TP, Franca-Silva MS, et al. (2010) Superoxide scavenging in the rostral ventrolateral medulla blunts the pressor response to peripheral chemoreflex activation. Brain Res 1351, 141-149.

19. Chan SH \& Chan JY (2012) Brain stem oxidative stress and its associated signaling in the regulation of sympathetic vasomotor tone. J Appl Physiol (1985) 113, 1921-1928.

20. Chobanyan-Jurgens K \& Jordan J (2015) Autonomic nervous system activity and inflammation: good ideas, good treatments, or both? Am J Physiol Heart Circ Physiol 309, H1999-H2001.

21. Simms AE, Paton JF, Pickering AE, et al. (2009) Amplified respiratory-sympathetic coupling in the spontaneously hypertensive rat: does it contribute to hypertension? J Physiol 587, 597-610.
22. Folkow B (1989) Sympathetic nervous control of blood pressure Role in primary hypertension. Am J Hypertens 2, 103S-111S.

23. Mancia G, Bousquet P, Elghozi JL, et al. (2007) The sympathetic nervous system and the metabolic syndrome. J Hyperten 25, 909-920.

24. Cruz JC, Flor AF, Franca-Silva MS, et al. (2015) Reactive oxygen species in the paraventricular nucleus of the hypothalamus alter sympathetic activity during metabolic syndrome. Front Physiol 6, 384.

25. Corry DB \& Tuck ML (1999) Obesity, hypertension, and sympathetic nervous system activity. Curr Hypertens Rep 1, 119-126.

26. Franchini KG \& Krieger EM (1993) Cardiovascular responses of conscious rats to carotid body chemoreceptor stimulation by intravenous KCN. J Auton Nerv Syst 42, 63-69.

27. Zoccal DB, Simms AE, Bonagamba LG, et al. (2008) Increased sympathetic outflow in juvenile rats submitted to chronic intermittent hypoxia correlates with enhanced expiratory activity. J Physiol 586, 3253-3265.

28. Bayne K (1996) Revised guide for the care and use of laboratory animals available. American Physiological Society. Physiologist 39, 199, 208-111.

29. Reeves PG, Nielsen FH \& Fahey GC Jr (1993) AIN-93 purified diets for laboratory rodents: final report of the American Institute of Nutrition ad hoc writing committee on the reformulation of the AIN-76A rodent diet. J Nutr 123, 1939-1951.

30. de Brito Alves JL, Nogueira VO, Cavalcanti Neto MP, et al. (2015) Maternal protein restriction increases respiratory and sympathetic activities and sensitizes peripheral chemoreflex in male rat offspring. J Nutr 145, 907-914.

31. Traill MA (1973) Estimation of the concentration of low density lipoprotein cholesterol and very low density lipoprotein cholesterol by selective adsorption from serum with magnesium compounds. Lab Pract 22, 527-529.

32. Mehlem A, Hagberg CE, Muhl L, et al. (2013) Imaging of neutral lipids by oil red $\mathrm{O}$ for analyzing the metabolic status in health and disease. Nat Protoc 8, 1149-1154.

33. Cavalcanti CO, Alves RR, de Oliveira AL, et al. (2016) Inhibition of PDE5 restores depressed baroreflex sensitivity in renovascular hypertensive rats. Front Physiol 7, 15.

34. Malan A (1973) Ventilation measured by body plethysmography in hibernating mammals and in poikilotherms. Respir Physiol 17, 32-44.

35. El-Sayyad HI, Al-Haggar MM, El-Ghawet HA, et al. (2014) Effect of maternal diabetes and hypercholesterolemia on fetal liver of albino Wistar rats. Nutrition 30, 326-336.

36. Tsuduki T, Yamamoto K, Hatakeyama Y, et al. (2016) High dietary cholesterol intake during lactation promotes development of fatty liver in offspring of mice. Mol Nutr Food Res 60, 1110-1117.

37. Sun B, Purcell RH, Terrillion CE, et al. (2012) Maternal high-fat diet during gestation or suckling differentially affects offspring leptin sensitivity and obesity. Diabetes 61, 2833-2841.

38. De Assis SM, Seguro AC \& Helou CM (2003) Effects of maternal hypercholesterolemia on pregnancy and development of offspring. Pediatr Nephrol 18, 328-334.

39. Simms AE, Paton JF, Pickering AE, et al. (2009) Amplified respiratory-sympathetic coupling in the spontaneously hypertensive rat: does it contribute to hypertension? J Physiol 587, 597-610.

40. Grassi G (1998) Role of the sympathetic nervous system in human hypertension. J Hypertens 16, 1979-1987.

41. Barros MA, De Brito Alves JL, Nogueira VO, et al. (2015) Maternal low-protein diet induces changes in the cardiovascular autonomic modulation in male rat offspring. Nutr Metab Cardiovasc Dis 25, 123-130.

42. Carthy ER (2014) Autonomic dysfunction in essential hypertension: a systematic review. Ann Med Surg (Lond) 3, 2-7. 
43. Meguro Y, Miura Y, Kimura S, et al. (1989) A sympathetic component of baroreflex function in patients with essential hypertension. Clin Exp Pharmacol Physiol 15, 93-95.

44. Dempsey JA, Sheel AW, St Croix CM, et al. (2002) Respiratory influences on sympathetic vasomotor outflow in humans. Respir Physiol Neurobiol 130, 3-20.

45. Prabhakar NR (2013) Sensing hypoxia: physiology, genetics and epigenetics. J Physiol 591, 2245-2257.

46. Bavis RW \& MacFarlane PM (2017) Developmental plasticity in the neural control of breathing. Exp Neurol 287, 176-191.

47. Hill CB, Grandgeorge SH \& Bavis RW (2013) Developmental hyperoxia alters CNS mechanisms underlying hypoxic ventilatory depression in neonatal rats. Respir Physiol Neurobiol 189, 498-505.

48. Zambrano E, Ibanez C, Martinez-Samayoa PM, et al. (2016) Maternal obesity: lifelong metabolic outcomes for offspring from poor developmental trajectories during the perinatal period. Arch Med Res 47, 1-12.

49. Myatt L \& Maloyan A (2016) Obesity and placental function. Semin Reprod Med 34, 42-49.

50. Samarasinghe TD, Sands SA, Skuza EM, et al. (2015) The effect of prenatal maternal infection on respiratory function in mouse offspring: evidence for enhanced chemosensitivity. $J$ Appl Physiol (1985) 119, 299-307.

51. Forsberg D, Horn Z, Tserga E, et al. (2016) $\mathrm{CO}_{2}$-evoked release of $\mathrm{PGE}_{2}$ modulates sighs and inspiration as demonstrated in brainstem organotypic culture. elife $\mathbf{5}$, $1-41$.

52. Bayol SA, Farrington SJ \& Stickland NC (2007) A maternal 'junk food' diet in pregnancy and lactation promotes an exacerbated taste for 'junk food' and a greater propensity for obesity in rat offspring. Br J Nutr 98, 843-851. 